\title{
Immunogenicity of the Spike Glycoprotein of Bat SARS-like Coronavirus*
}

\author{
Yu-xuan HOU ${ }^{1}$, Cheng PENG ${ }^{1}$, Zheng-gang HAN ${ }^{1}$, Peng ZHOU ${ }^{1}$, \\ Ji-guo $\mathrm{CHEN}^{2}$ and Zheng-li SHI ${ }^{\text {*** }}$
}

(1. State Key Laboratory of Virology, Wuhan Institute of Virology, Chinese Academy of Sciences, Wuhan 430071, China; 2. Department of Biological Sciences, Mississippi State University, Mississippi State, MS 39762, USA)

\begin{abstract}
A group of SARS-like coronaviruses (SL-CoV) have been identified in horseshoe bats. Despite SL-CoVs and SARS-CoV share identical genome structure and high-level sequence similarity, SL-CoV does not bind to the same cellular receptor as for SARS-CoV and the N-terminus of the S proteins only share $64 \%$ amino acid identity, suggesting there are fundamental differences between these two groups of coronaviruses. To gain insight into the basis of this difference, we established a recombinant adenovirus system expressing the $\mathrm{S}$ protein from SL-CoV (rAd-Rp3-S) to investigate its immune characterization. Our results showed that immunized mice generated strong humoral immune responses against the SL-CoV S protein. Moreover, a strong cellular immune response demonstrated by elevated IFN- $\gamma$ and IL- 6 levels was also observed in these mice. However, the induced antibody from these mice had weaker cross-reaction with the SARS-CoV S protein, and did not neutralize HIV pseudotyped with SARS-CoV S protein. These results demonstrated that the immunogenicity of the SL-CoV S protein is distinct from that of SARS-CoV, which may cause the immunological differences between human SARS-CoV and bat SL-CoV. Furthermore, the recombinant virus could serve as a potential vaccine candidate against bat SL-CoV infection.
\end{abstract}

Key words: SARS coronavirus (SARS-CoV); SARS-like coronavirus (SL-CoV); Spike glycoprotein; Humoral immune response; Cellular immune response

Severe acute respiratory syndrome coronavirus (SARS-CoV) is the etiological agent of SARS which emerged in Guangdong province, China during 2002 and $2003^{[7,17,22,23,27,36]}$. Closely related viruses were

Received: 2009-08-24, Accepted:2009-10-28

* Foundation items: This work was supported by the State Key Program for Basic Research Grant (2005CB523004) from the Chinese Ministry of Science and Technology, the Knowledge Innovation Program Key Project administered by the Chinese Academy of Sciences (KSCX1-YW-R-07).

** Corresponding author.

Phone/Fax:+86-27-87197240, E-mail: zlshi@wh.iov.cn identified in masked palm civets (Paguma larvata), raccoon dogs (Neyctereutes procyonoides) in wild markets, suggesting that SARS-CoV is a zoonotic virus which was recently transmitted to human ${ }^{[9]}$. Subsequently, a group of genetically diverse CoVs, named SARS-like CoV (SL-CoV), was discovered in horseshoe bats (genus Rhinolophus), further demonstrated that bats may be natural reservoirs of SARS-CoV ${ }^{[18,20]}$.

Bat SL-CoV and human SARS-CoV share an identical 
genome organization and high sequence identities in gene products (92\%-100\%) with the main exception of the N-terminus of the spike glycoprotein (S protein) which shares only $64 \%$ amino acid sequence identity, implying that the bat SL-CoV S protein has different properties from that of SARS-CoV ${ }^{[20]}$. The $\mathrm{S}$ protein of SARS-CoV is responsible for binding the cellular receptor, angiotensin-converting enzyme 2 (ACE2) ${ }^{[19]}$, and mediates viral entry ${ }^{[6,16,28]}$. Additionally, the $\mathrm{S}$ protein induces protective humoral and cellular responses in the host $\mathrm{t}^{[4,5,10,11,13-15,21,32,34,37]}$. For the bat SL-CoV S protein, by using the HIV-pseudotyped SARS-CoV and SL-CoV S protein, we have demonstrated that SL-CoV could not use ACE2 as its receptor. However, the replacement of the SARS-CoV receptor binding domain (RBD) to SL-CoV S protein could convert the SL-CoV pseudovirus to be susceptible to the human ACE2 expressing cells ${ }^{[26]}$. Furthermore, our previous study showed that viral-like particles containing the SL-CoV S protein have a stronger ability to stimulate dendritric cells (DCs) to cytokine induction than those containing the SARS-CoV S protein. And SL-CoV S DNA vaccine evoked a more vigorous antibody response and a stronger $\mathrm{T}$ cell response than the SARS-CoV S DNA in mice ${ }^{[1]}$. These results showed that the SL-CoV S protein has properties that are distinct from SARS-CoV S protein. Thus it is of great importance to elucidate its immunogenicity.

In this study, we constructed a recombinant adenovirus expressing the full-length codon-optimized $\mathrm{S}$ gene of bat SL-CoV Rp3 isolate (rAd-Rp3-S), and investigated its ability to induce humoral and cellular immune responses in mice. The ELISA data showed that the rAd-Rp3-S immunized mice generated strong humoral immune responses against the HIV- pseudotyped SL-CoV S protein. However, the induced antibody had weaker cross-reaction with the HIV-pseudotyped SARS-CoV $S$ protein; neither neutralized the pseudovirus. ELISPOT assay found that the secretion levels of IFN- $\gamma$ and IL-6 in the rAd-Rp3-S immunized mice were higher than that of the negative control, demonstrating that the cellular immune responses were also elicited in the rAd-Rp3-S immunized mice. Our study demonstrated that the $\mathrm{S}$ protein from bat SL-CoV can elicit effective immune responses. It also suggested that rAd-Rp3-S can be a potential vaccine candidate against the group of SL-CoVs in the future.

\section{MATERIALS AND METHODS}

\section{Construction and purification of rAd-Rp3-S}

The AdEasy adenoviral vector system (Stratagene), comprising the shuttle vector pAdTrack-CMV and the backbone vector pAdEasy-1, was used to construct a recombinant adenovirus carrying the full length codon-optimized S gene of bat SL-CoV Rp3 isolate (GenBank accession NO.DQ071615) ${ }^{[26]}$, named rAd-Rp3-S. Briefly, the full-length codon-optimized S gene of bat SL-CoV Rp3 isolate was amplified from the plasmid pcDNA3.1-Rp3-S with primers 5'-GTA GTCGACACCATGGACGCCATGAAGAGGGG-3', and 5'-GTACTCGAGTCATCTTTTCTCAGCCAT CGC-3' (Sal I and Xho I sites are underlined) and subcloned to pAdTrack-CMV through Sal I and Xho I sites. Secondly, pAdTrack-CMV carrying the $\mathrm{S}$ gene was digested by Pme I, homo recombined with pAdEasy-1 to generate pAd- Rp3-S. Lastly, rAdRp3-S was obtained by transfecting PacI-linearized pAd- Rp3-S into 293 cells. In parallel, rAd-GFP, a recombinant adenoviral vector containing the GFP 
gene, was constructed according to the same procedure and used as vector control. Both of the two recombinant adenoviruses were observed under florescent microscopy and titered with GFP marker. The virus was purified by CsCl-banding and observed under electronic microscopy (EM).

\section{Reverse transcription-polymerase chain reaction (RT-PCR) analysis}

293 cells were maintained in DMEM supplemented with $100 \mathrm{IU} / \mathrm{mL}$ of penicillin and $100 \mu \mathrm{g} / \mathrm{mL}$ of streptomycin and 10\% fetal calf serum. To detect S gene transcriptional level, total RNA of 293 cells infected by recombinant adenoviruses were extracted using TRIZOL ${ }^{\circledR}$ reagent (Invitrogen), then treated with DNase I (RNase free) at $37^{\circ} \mathrm{C}$ for 30 mins. The first strand cDNAs were synthesized with MLV Reverse Transcriptase with random primers (Promega). Subsequent PCR was performed with primers specific for the codon-optimized S gene of Rp3, Rp3SF (5'-C GCGGATCCACCATGGCCCAGGAGGGCTGC-3’) and Rp3SR (5'-CCGGAATTCGAGACATAGTGTA AGCCAC-3') to generate a $1956 \mathrm{bp}$ fragment. The $\beta$-actin was amplified with primers AF (5-TGGCG CTTTTGACTCAGGAT-3) and AR (5'-AGCCCTG GCTGCCTCAAC-3') to produce a $450 \mathrm{bp}$ fragment that was assayed as an internal control. RT-PCR products were analyzed by $0.8 \%$ agarose gel.

\section{Western blot analysis}

293 cells were harvested at $48 \mathrm{~h}$ post infection and lysed in SDS buffer $(50 \mathrm{mmol} / \mathrm{L}$ Tris. $\mathrm{HCl}$, pH6.8, $100 \mathrm{mmol} / \mathrm{L}$ DTT, 2\% SDS, 0.1\% bromophenol blue, $10 \%$ glycerol). Lysate protein were fractionated on 4\%-10\% SDS-PAGE and transferred to polyvinylidene difluoride (PVDF) by semi-dry protein transfer apparatus (Bio-Rad, Hercules, CA).The membrane was blocked for $2 \mathrm{~h}$ with $3 \%$ skimmed milk in TBS buffer $(20 \mathrm{mmol} / \mathrm{L}$ Tris base, $137 \mathrm{mmol} / \mathrm{L} \mathrm{NaCl}, \mathrm{pH}$ 7.6). Following, the blocked membrane was probed with F26G8, mouse IgG mono-antibody against the SARS-CoV S protein that was kindly provided by Dr. Berry $^{[3]}$ and bound with goat anti-mouse AP-conjugated antibody (Santa Cruz Biotechnologies, Santa Cruz, CA). The results were finally revealed by using chemical substrate NBT and BCIP coloration.

\section{Immunization of mice}

The six-week-old female BALB/c mice $(n=5$ per group) were intraperitoneally immunized every 2 weeks with rAd-Rp3-S $\left(1 \times 10^{7}\right.$ pfu per dose $)$ or with the negative controls (rAd-GFP or PBS). Mice were immunized three times for each condition before bleeding.

\section{Enzyme-linked immunosorbent assay (ELISA)}

Sera from the immunized mice of different groups were collected on day $0,14,28$ and $42 \mathrm{~d}$. S protein specific antibodies (IgGs) were titered by ELISA. Firstly, HIV/BJ01-S and HIV/Rp3-S, pseudotyped with SARS-CoV (BJ01 isolate, AY278488) and SLCoV (Rp3 isolate, DQ071615) S proteins, were produced as described previously ${ }^{[26]}$. The two pseudoviruses were used antigens and coated in separate 96-well microtiter plates. Then, the plates were incubated at $4{ }^{\circ} \mathrm{C}$ overnight and blocked with PBS (pH 7.4) containing 3\% skimmed milk at $37^{\circ} \mathrm{C}$ for $2 \mathrm{~h}$. Mouse sera, diluted 500-fold, were added to the wells and incubated at $37^{\circ} \mathrm{C}$ for $1 \mathrm{~h}$. Wells were rinsed five times, 3 min per time with PBST (PBS containing $0.05 \%$ Tween-20), followed by incubation with horseradish- peroxidase -conjugated goat-antimouse IgG (Sigma) $(1: 5000)$ at $37^{\circ} \mathrm{C}$ for $45 \mathrm{~min}$ and a subsequent $15 \mathrm{~min}$ incubation with the substrate 
TMB solution at room temperature in dark. Reactions were terminated with a stop solution, and optical densities $(O D)$ were determined using a microplate reader set at $450 \mathrm{~nm}$.

\section{Sera neutralization assay}

Pseudovirus-based neutralization assay was used to determine neutralization ability of immunized sera to HIV/BJ01-S. The neutralizing activity of heatinactivated sera $\left(56^{\circ} \mathrm{C}, 30 \mathrm{~min}\right)$ was determined by mixing $10 \mathrm{ng}$ of pseudovirues (in $30 \mu \mathrm{L}$ ) with diluted antisera (in $30 \mu \mathrm{L}$ ) at $37{ }^{\circ} \mathrm{C}$ for $1 \mathrm{~h}$. Antiserapseudovirus complexes were then mixed with $16 \mathrm{ng}$ polybrene (in $40 \mu \mathrm{L}$ medium) before they were added to human ACE2 expressing HeLa cells. The infected cells were washed with PBS and lysed (Cell Culture Lysis Reagent, Promega) at $48 \mathrm{~h}$ post infection. The neutralization activity of each antiserum was monitored by measurement of luciferase intensity as described previously ${ }^{[26]}$. Due to a lack of permissive cell lines for bat SARS-like CoV, the sera neutralization assay to this virus was not conducted.

\section{ELISPOT assay}

To test cellular immune responses in mice, ELISPOT assays were performed to detect the secretion levels of IFN- $\gamma$ and IL-6 following the protocol of the manufacturer (U-CyTech, Netherlands; eBioscience, USA). Briefly, 96-well a ELISPOT plate was coated with $100 \mu \mathrm{L}$ anti-mouse IFN- $\gamma$ or IL-6 capture antibody solution per well and left overnight at $4^{\circ} \mathrm{C}$. The next day, wells were washed and blocked with complete RPMI-1640 at room temperature for $2 \mathrm{~h}$. Splenocytes $\left(2 \times 10^{5}\right.$ per cell) from mice immunized for 56 days were added to microwells along with purified Rp3-S protein $(10 \mu \mathrm{g} / \mathrm{mL})$ as the specific stimulating antigen. Cells were incubated at $37^{\circ} \mathrm{C}$ and $5 \% \mathrm{CO}_{2}$ for $36 \mathrm{~h}$. Then wells were extensively washed with PBS containing $0.05 \%$ Tween 20 and subsequently incubated with biotin-conjugate anti-mouse IFN- $\gamma$ or IL-6 detection antibody solution at room temperature for $2 \mathrm{~h}$. After washing, wells were incubated with streptavidin-horseradish peroxidase antibody solution at room temperature for $45 \mathrm{~min}$. Wells were washed again, and the freshly-prepared AEC substrate solution was added to wells. Color development was monitored for 10-60 min and stopped by washing with distilled water. After air-drying the plate at room temperature, wells were counted using an ELISPOT reader (Hitech Instruments).

\section{RESULTS}

\section{Characterization of rAd-Rp3-S}

The recombinant adenovirus, rAd-Rp3-S was observed by EM. The recombinant virus displayed a typical morphology characteristic of adenovirus (data not shown). The titers of rAd-Rp3-S and rAd-GFP, determined by GFP fluorencence, were up to $6.45 \times 10^{7} \mathrm{PFU} / \mathrm{mL}$ and $5.53 \times 10^{7} \mathrm{PFU} / \mathrm{mL}$, respectively. Expression of S protein in rAd-Rp3-S infected 293 cells

Green fluorescence was observed in rAd-Rp3-S infected cells at $24 \mathrm{~h}$ post infection. The RT-PCR result showed that an expected 1956bp fragment of bat SL-CoV S gene was obtained only in 293 cells infected by rAd-Rp3-S (Fig. 1A). An expected 450 bp fragment specific for $\beta$-actin was produced in 293 cells infected by rAd-Rp3-S and rAd-GFP (Fig. 1A). For westernblot, an expected $150-200 \mathrm{kDa}$ fragment that was in agreement with the size of SL-CoV S protein was observed in 293 cells infected by rAd- Rp3-S, but was not found in cells infected by rAd-GFP (Fig. 1B). 
A

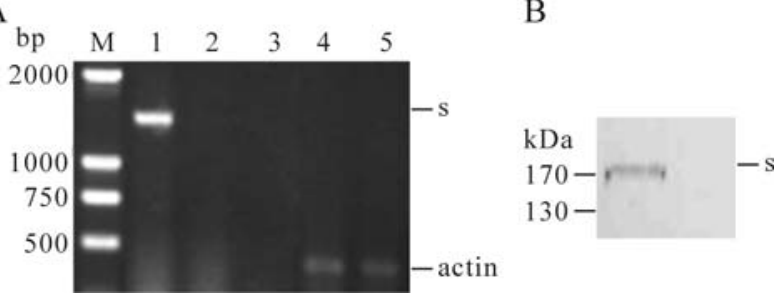

Fig. 1. Expression of bat SL-CoV S protein in rAd-Rp3-S infected 293 cells. A: 293 cells were infected with rAd-Rp3-S and rAd-GFP respectively for $48 \mathrm{~h}$ and total RNAs were extracted for RT-PCR analysis. Lane 1 and 4, 293 cells infected with rAd -Rp3-S; Lane 2 and 5, 293 cells infected with rAd -GFP; Lane 3, the distilled water was as the blank control. B: 293 cells were infected with rAd-Rp3-S and rAd-GFP respectively for $48 \mathrm{~h}$, the infected cells were collected and lysed for western blot analysis. Lane 1, 293 cells infected with rAd-Rp3-S; Lane 2, 293 cells infected with rAd -GFP. Arrow indicates the specific $\mathrm{S}$ protein.

\section{Specific antibody responses in rAd-Rp3-S immunized} mice

The mouse sera collected on day $0,14,28$, and 42 was used to detect specific antibody responses against HIV/BJ01-S and HIV/Rp3-S. ELISA results showed that the specific antibody against HIV/BJ01-S or HIV/ Rp3-S was detected in rAd-Rp3-S immunized mouse sera on the $14^{\text {th }}$ day, the titer boosted dramatically after day 28 and was 10-fold higher than that in rAdGFP immunized mice by day 42 ; no specific antibody

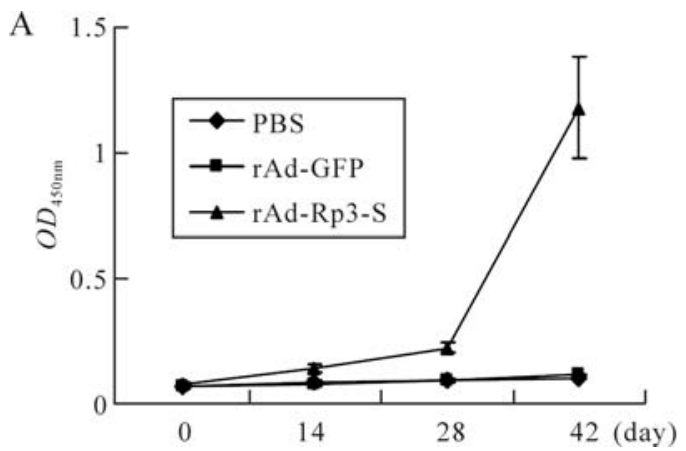

responses were found at detectable levels in negative control groups, rAd-GFP or PBS immunized mice (Fig. 2). Further, the antibody titer against HIV/Rp3-S was much higher than that against HIV/BJ01-S in rAd-Rp3-S immunized mice. These results indicated that the specific antibody against SL- CoV S protein and the weaker cross with SARS-CoV S protein were generated in rAd-Rp3-S immunized mice.

Due to lack of permissive cell lines for SL-CoV, HIV/BJ01-S which has a similar infectivity to wild SARS-CoV ${ }^{[26]}$, was used to detect the neutralization of antibodies induced by rAd-Rp3-S. The cells infected by mixtures of HIV/BJ01-S and mouse sera induced by rAd-Rp3-S, rAd-GFP and PBS did not afford any protection. However, high neutralizing activity was found in mouse sera induced by the full length SARS-CoV S protein (BJ01-S) (Fig. 3). The serum neutralization assay demonstrated that the antibody induced by bat SL-CoV S protein couldn't neutralize the HIV/BJ01-S and so the recombinant adenovirus rAd-Rp3-S may not be a cross protective candidate for SARS-CoV.

\section{The cellular immune responses in rAd-Rp3-S immunized mice}

B

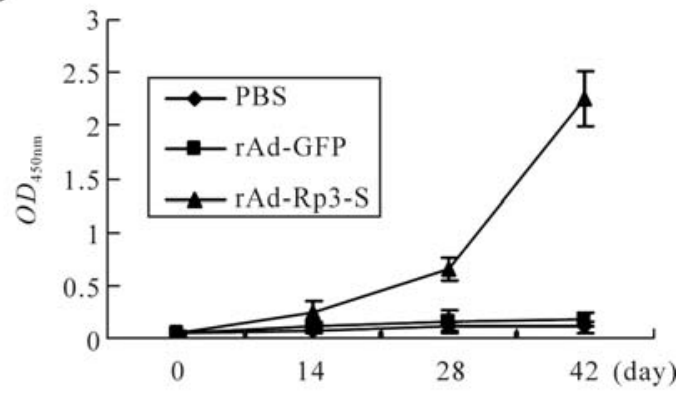

Fig. 2. ELISA detection of specific antibody against bat SL-CoV S protein in immunized mice. Mice were immunized with, rAd-Rp3-S, rAd-GFP or PBS. Sera of immunized mice from different groups were collected on day 0, 14, 28, 42. The titers of antibodies against pseudovirus HIV/BJ01-S (A) and HIV/Rp3-S (B) were measured by ELISA. Data shown are the mean \pm S.D. of three experiments from the different group. 


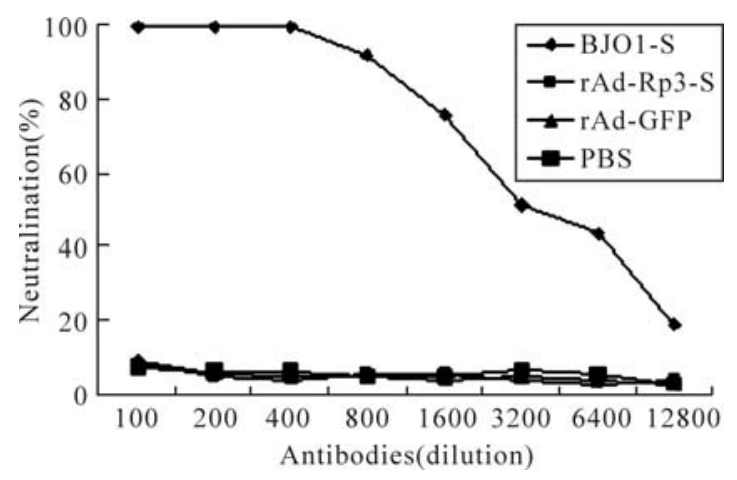

Fig. 3. Neutralization test of antibody induced by rAd-Rp3-S. The antibody against BJ01-S, rAd-Rp3-S or rAd-GFP (dilution at 1: 100 to 1:12 800) was mixed with HIV/BJ01-S and tested the infectivity to HEK293 cells expressing human ACE2. Percentage of neutralization activity was calculated for each antiserum as described in reference ${ }^{[26]}$, and the average values were plotted.

The splenocytes of the immunized mice that were harvested on the eighth week were used to detect the secretion levels of IFN- $\gamma$ and IL-6 by ELISPOT assay. The secretion numbers of IFN- $\gamma$ were about 120 cells per $10^{6}$ cells in rAd-Rp3-S immunized mouse splenocytes. However, the secretion numbers of IFN- $\gamma$ were less than 10 cells per $10^{6}$ cells in Ad-GFP or PBS immunized mouse (Fig. 4A). Similar to IFN- $\gamma$, the secretion numbers of IL-6 were up to about 160 cells per $10^{6}$ cells in rAd-Rp3-S immunized mouse splenocytes, while only about 60 IL- 6 secretion cells per $10^{6}$ cells in Ad-GFP or PBS immunized mouse (Fig. 4B).
These data demonstrated that IFN- $\gamma$, Th1 cytokine, and IL-6, Th2 cytokine, were both elicited in the rAdRp3-S immunized mouse and bat SL-CoV S protein can generate specific cellular immune responses in immunized mice.

\section{DISCUSSION}

The discovery of genetic diverse bat SL-CoVs in nature poses a potential threat to domestic animals and humans. However, little is known about this group of viruses, for example, the biological property, pathogenesis and immunogenicity. In this study, we successfully constructed a recombinant adenovirus containing the full length codon-optimized S gene of the bat SL-CoV. The recombinant virus, rAd-Rp3-S, elicited strong antibody response in mice against SLCoV S protein, but a low cross reaction with SARS$\mathrm{CoV}$ (BJ01 isolate) $\mathrm{S}$ protein and failed to neutralize the HIV/BJ01-S protein. These results indicate that the recombinant virus can induce a specific antibody response against bat SL-CoV S protein in a mice model and the bat SL-CoV S protein has different immune responses from that of SARS-CoV. These results are consistent with our previous results that bat sera naturally infected by SL-CoV could not neutralize the human SARS-CoV ${ }^{[20]}$.
A

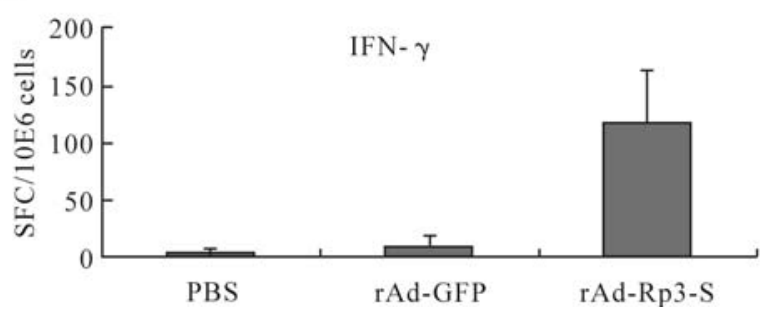

B

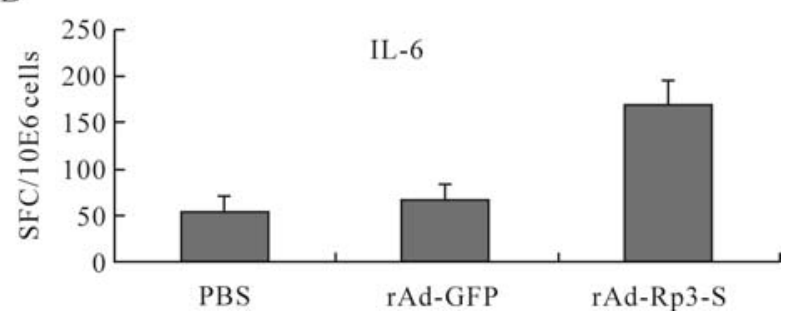

Fig. 4. ELISPOT detection of cytokine in immunized mice. The secretion levels of IFN- $\gamma$ (A) and IL-6 (B) were detected in mouse splenocytes immunized by rAd-Rp3-S, rAd-GFP or PBS on day 56 post immunization. Data shown are the mean \pm S.D. of three experiments from the different group. SFC means spot forming cell. 
The RBD of SARS-CoV $\mathrm{S}$ protein contains neutralizing epitopes and can induce neutralizing antibodies $^{[5,10,13,14]}$. The minor mutations in the $\mathrm{S}$ protein, particularly the receptor binding domain (RBD), abolished viral entry into the susceptible cells and failed to induce cross-protection neutralizing antibodies $^{[12,35]}$. An important difference between the bat SL-CoV and SARS-CoV S proteins occurs in the RBD, the RBD of bat SL-CoV S protein has two deletions (5 and 12-13 aa, respectively). This important difference led to different usage of the cellular receptor between SARS-CoV and SL-CoV; the bat SL-CoV cannot use ACE2 as its receptor ${ }^{[26]}$. Our current study further demonstrated that the divergence between the SARS-CoV and SL-CoV S protein also may cause the antibody induced by SL-CoV S protein to have a weaker cross reaction with SARS-CoV S protein and such that it did not neutralize HIV/ BJ01-S.

Cellular immune responses are very important for host defense against the viral infection. IFN- $\gamma$ is synthesized almost exclusively by activated natural killer (NK) and T cells in response to viral infection ${ }^{[24]}$. IL-6 plays a central role in both innate and acquired immune response. In our study, high secretion level of IFN- $\gamma\left(120\right.$ cells per $10^{6}$ cells) and IL-6 (160 cells per $10^{6}$ cells) in rAd-Rp3-S immunized mice splenocytes, indicated that both Th1 and Th2 cells were elicited in rAd-Rp3-S immunized mice. Similar responses have been observed in SARS patients and animal models, for example, the level of IFN- $\gamma$ in SARS patients at the early stages of disease onset correlated with resolution of the viral infection ${ }^{[29]}$, and IL-6 was up-regulated as a consequence of being induced by SARS-CoV S protein in murine macrophages ${ }^{[31]}$.
It is clear that the recently discovered bat SL-CoVs are not the immediate progenitors of SARS-CoV which caused the outbreaks in $2002 / 2003^{[26]}$. It is envisaged that the ongoing vaccine against the SARS$\mathrm{CoV}$ may not protect the infection by bat SL-CoVs. Considering the wide distribution and genetic diversity of bat SL-CoVs in China ${ }^{[18,20,25,33]}$, co-infection of same bat species by different coronaviruses ${ }^{[18,20,30]}$ and the capability of recombination of coronaviruses ${ }^{[2,8]}$, it is likely new coronaviruses in bats that can cross species to infect humans. Moreover, high density of bat habitats and increasing contacts between bats and human will further increase the virus transmission opportunities from bats to human. So, it is highly important to be prepared for prevention and control of the emerging disease resulting from this group of viruses. The recombinant adenovirus constructed in this study provides a potential vaccine candidate against the infection by diverse bat SL-CoVs.

\section{Acknowledgements}

We thank Dr. Ying Zhu of Wuhan University, China for kindly providing AdEasy adenoviral system. We thank Xuefang An of Wuhan Institute of virology, Chinese Academy of Sciences, China, for technical help of mice immunization. This work was jointly funded by the State Key Program for Basic Research Grant (2005CB523004) from the Chinese Ministry of Science and Technology, the Knowledge Innovation Program Key Project administered by the Chinese Academy of Sciences (KSCX1-YW-R-07).

\section{References}

1. Bai B, Hu Q, Hu H, et al. 2008. Virus-like particles of SARS-like coronavirus formed by membrane proteins 
from different origins demonstrate stimulating activity in human dendritic cells. PLoS ONE, 3: e2685.

2. Baric R S, Fu K, Schaad, M C, et al. 1990. Establishing a genetic recombination map for murine coronavirus strain A59 complementation groups. Virology, 177: 646656.

3. Berry J D, Jones S, Drebot M A, et al. 2004. Development and characterisation of neutralising monoclonal antibody to the SARS-coronavirus. J Virol Methods, 120: 87-96.

4. Bisht H, Roberts A, Vogel L, et al. 2005. Neutralizing antibody and protective immunity to SARS coronavirus infection of mice induced by a soluble recombinant polypeptide containing an N-terminal segment of the spike glycoprotein. Virology, 334: 160-165.

5. Chen Z, Zhang L, Qin C, et al. 2005. Recombinant modified vaccinia virus Ankara expressing the spike glycoprotein of severe acute respiratory syndrome coronavirus induces protective neutralizing antibodies primarily targeting the receptor binding region. J Virol, 79: $2678-2688$

6. Chou C F, Shen S, Tan Y J, et al. 2005. A novel cell-based binding assay system reconstituting interaction between SARS-CoV S protein and its cellular receptor. J Virol Methods, 123: 41-48.

7. Fouchier R A, Kuiken T, Schutten M, et al. 2003. Aetiology: Koch's postulates fulfilled for SARS virus. Nature, 423:240.

8. Fu K,Baric R S. 1992. Evidence for variable rates of recombination in the MHV genome. Virology, 189: 88-102.

9. Guan Y, Zheng B J, He Y Q, et al. 2003. Isolation and characterization of viruses related to the SARS coronavirus from animals in southern China. Science, $302: 276-278$

10. He Y, Li J, Du L, et al. 2006. Identification and characterization of novel neutralizing epitopes in the receptor-binding domain of SARS-CoV spike protein: revealing the critical antigenic determinants in inactivated SARS-CoV vaccine. Vaccine, 24: 5498-5508.

11. He Y, Li J, Heck S, et al. 2006. Antigenic and immunogenic characterization of recombinant baculovirusexpressed severe acute respiratory syndrome coronavirus spike protein: implication for vaccine design. J Virol, 80: $5757-5767$
12. He Y, Li J, Jiang S. 2006. A single amino acid substitution (R441A) in the receptor-binding domain of SARS coronavirus spike protein disrupts the antigenic structure and binding activity. Biochem Biophys Res Commun, 344: 106-113.

13. He Y, Lu H, Siddiqui P, et al. 2005. Receptor-binding domain of severe acute respiratory syndrome coronavirus spike protein contains multiple conformation-dependent epitopes that induce highly potent neutralizing antibodies.

J Immunol, 174: 4908-4915.

14. He Y, Zhou Y, Liu S, et al. 2004. Receptor-binding domain of SARS-CoV spike protein induces highly potent neutralizing antibodies: implication for developing subunit vaccine. Biochem Biophys Res Commun, 324: 773-781.

15. He Y, Zhou Y, Wu H, et al. 2004. Identification of immunodominant sites on the spike protein of severe acute respiratory syndrome (SARS) coronavirus: implication for developing SARS diagnostics and vaccines. J Immunol, 173: 4050-4057.

16. Ho T Y, Wu S L, Cheng S E, et al. 2004. Antigenicity and receptor-binding ability of recombinant SARS coronavirus spike protein. Biochem Biophys Res Commun, 313: 938-947.

17. Ksiazek T G, Erdman D, Goldsmith C S, et al. 2003. A novel coronavirus associated with severe acute respiratory syndrome. N Engl J Med, 348: 1953-1966.

18. Lau S K, Woo P C, Li K S, et al. 2005. Severe acute respiratory syndrome coronavirus-like virus in Chinese horseshoe bats. Proc Natl Acad Sci U S A, 102: 1404014045.

19. Li W, Moore M J, Vasilieva N, et al. 2003. Angiotensin-converting enzyme 2 is a functional receptor for the SARS coronavirus. Nature, 426: 450-454.

20. Li W, Shi Z, Yu M, et al. 2005. Bats are natural reservoirs of SARS-like coronaviruses. Science, 310: 676-679.

21. Liu R Y, Wu L Z, Huang B J, et al. 2005. Adenoviral expression of a truncated S1 subunit of SARS-CoV spike protein results in specific humoral immune responses against SARS-CoV in rats. Virus Res, 112: 24-31.

22. Marra M A, Jones S J, Astell C R, et al. 2003. The Genome sequence of the SARS-associated coronavirus. Science, 300: 1399-1404. 
23. Peiris J S, Yuen K Y, Osterhaus A D, et al. 2003. The severe acute respiratory syndrome. N Engl J Med, 349: 2431-2441.

24. Pfeffer L M, Dinarello C A, Herberman R B, et al. 1998. Biological properties of recombinant alphainterferons: 40th anniversary of the discovery of interferons. Cancer Res, 58: 2489-2499.

25. Poon L L, Chu D K, Chan K H, et al. 2005. Identification of a novel coronavirus in bats. J Virol, 79: 2001-2009.

26. Ren W, Qu X, Li W, et al. 2008. Difference in receptor usage between severe acute respiratory syndrome (SARS) coronavirus and SARS-like coronavirus of bat origin. $\mathbf{J}$ Virol, 82: 1899-1907.

27. Rota P A, Oberste M S, Monroe S S, et al. 2003. Characterization of a novel coronavirus associated with severe acute respiratory syndrome. Science, 300: 13941399.

28. Simmons G, Reeves J D, Rennekamp A J, et al. 2004. Characterization of severe acute respiratory syndromeassociated coronavirus (SARS-CoV) spike glycoproteinmediated viral entry. Proc Natl Acad Sci USA, 101: $4240-4245$

29. Stroher U, DiCaro A, Li Y, et al. 2004. Severe acute respiratory syndrome-related coronavirus is inhibited by interferon-alpha. J Infect Dis, 189: 1164-1167.

30. Tang X C, Zhang J X, Zhang S Y, et al. 2006. Prevalence and genetic diversity of coronaviruses in bats from China. J Virol, 80 (15): 7481-7490.
31. Wang W, Ye L, Ye L, et al. 2007. Up-regulation of IL-6 and TNF-alpha induced by SARS-coronavirus spike protein in murine macrophages via NF-kappaB pathway. Virus Res, 128: 1-8.

32. Wang Y D, Sin W Y, Xu G B, et al. 2004. T-cell epitopes in severe acute respiratory syndrome (SARS) coronavirus spike protein elicit a specific T-cell immune response in patients who recover from SARS. J Virol, 78: $5612-5618$

33. Woo P C, Lau S K, Li K S, et al. 2006. Molecular diversity of coronaviruses in bats. Virology, 351: 180187.

34. Yang Z Y, Kong W P, Huang Y, et al. 2004. A DNA vaccine induces SARS coronavirus neutralization and protective immunity in mice. Nature, 428: 561-564.

35. Yi C E, Ba L, Zhang L, et al. 2005. Single amino acid substitutions in the severe acute respiratory syndrome coronavirus spike glycoprotein determine viral entry and immunogenicity of a major neutralizing domain. J Virol, 79: 11638-11646.

36. Zhong N S, Zheng B J, Li Y M, et al. 2003. Epidemiology and cause of severe acute respiratory syndrome (SARS) in Guangdong, People's Republic of China, in February, 2003. Lancet, 362: 1353-1358.

37. Zhou Z, Post P, Chubet R, et al. 2006. A recombinant baculovirus-expressed S glycoprotein vaccine elicits high titers of SARS-associated coronavirus (SARS-CoV) neutralizing antibodies in mice. Vaccine, 24: 3624-3631. 